\section{Bienenhonigallergie: Welches Allergen ist verantwortlich?}

M. Müller, M. Henzgen, C. Kroegel

Abt. Pneumologie \& Allergologie/Immunologie, Klinikum der FSU, Jena

Einleitung: Über Honigallergien finden sich in der Literatur nur gelegentlich Berichte. Meist sind Patienten mit schon bekannter Pollen- und Nahrungsmittelallergie betroffen. Verschiedene Kasuistiken beschreiben dabei Sensibilisierungen gegenüber Korbblütler-, Ahorn- und Rapspollen und zwei Kasuistiken eine Sensibilisierung gegenüber Bienenkörperbestandteilen als Ursache der Honigallergie.

Kasuistik: Wir berichten hier über eine 38-jährige Patientin, bei der es vor 18 Jahren erstmals nach Verzehr eines Honigbrötchens nach etwa 1 min zur Zungen- und Rachenschwellung sowie zu einem Angioödem der Augenumgebung, Übelkeit, Erbrechen und Durchfall kam. Bienenstiche in der Kindheit wurden mit ausgeprägter Lokalreaktion vertragen, in der Familienanamnese findet sich eine Bienengiftallergie bei der Großmutter mütterlicherseits. Eine Pollenallergie ist nicht bekannt.

Allergologische Diagnostik: Es konnte eine Sensibilisierung sowohl gegenüber Honig (spezifisches $\mathrm{IgE}$ deutlich positiv mit CAP-Klasse 4) als auch Bienengift (positiver Prick-Test, spezifisches IgE mit CAP-Klasse 2), jedoch nicht gegenüber Aeroallergenen nachgewiesen werden .

Therapieempfehlung: Eine Honigkarenz ist erforderlich und das Vorkommen auch als verstecktes Allergen in Nahrungsmitteln ist zu berücksichtigen. Aktuell besteht keine Indikation zur Bienengifthyposensibilisierung.

Diskussion: Im Bienenhonig finden sich neben den Hauptbestandteilen Zuckergemisch und Wasser u. a. auch Pollen, Pilze, Bakterien, Algen, Milben, Bienengift, Bienenkörperbestandteile sowie Körperteile von anderen Insekten. In unserem Fall ist am ehesten die gleichzeitig bestehende Bienengiftsensibilisierung als Auslöser der Nahrungsmittelunverträglichkeitsreaktion auf Honig anzusehen, eine Pollensensibilisierung wurde ausgeschlossen.

\section{Die Bedeutung des Immunblotting für die Diagnose von Insektengiftallergien}

K. Jung

Praxis für Dermatologie, Immunologie, Allergologie und Umweltmedizin, Erfurt

Die positive Anamnese und der Nachweis spezifischer IgEAntikörper (sIgE-Ak) sind erforderliche Kriterien für die Indikation einer spezifischen Immuntherapie (SIT) bei Insektengiftallergie. Aufgrund der Test-Sensitivitäten von 70-90\% an der Haut und in vitro wurde die Verwendbarkeit des Immunoblot-Systems AlaSTAT-AlaBLOT (DPC Biermann GmbH, Bad Nauheim) hinsichtlich des Nachweises sIgE-Ak gegen Hymenopterengiftallergene untersucht.

Bei 26 Patienten mit vorangegengenen systemischen Reaktionen nach Insektenstich wurde die Prick-Testung (ALK Scherax Arzneimittel GmbH, Hamburg) durchgeführt. Die Bestimmung der sIgE-Ak im Serum erfolgte mittels Magic
Lite SQ (Chirion, Fernwald) und dem Immunoblot-System AlaBLOT-AlaSTAT. Die Gruppe I bestand aus 5 Patienten mit Wespengiftallergie, Gruppe II aus 5 Patienten mit Bienengiftallergie. Gruppe III umfasste 6 Patienten mit Doppelsensibilisierung sowohl gegen Bienen- als auch Wespengift und 2 Patienten mit partiellen Kreuzreaktionen. In der Gruppe IV wurden 18 Patienten ohne Nachweis sIgE-Ak gegen Insektengift mittels Magic-Lite SQ untersucht.

Für die Gruppen I, II und III konnte eine gute Übereinstimmung zwischen gemessenen sIgE und semiquantitativoptisch bewerteten AlaSTAT-AlaBLOT fesgestellt werden. Es konnten jedoch in der Gruppe III keine spezifischen Immunoblot-Muster, die prophetisch auf eine Doppelsensibilisierung oder Kreuzreaktion hinweisen, bestimmt werden. Überraschend zeigten 8 Patienten der Gruppe IV Ak-Signale im Immunoblot. Sechs dieser Patienten besaßen einen positiven Prick-Test. Bei einem konnte wegen Urticaria factitia der Hauttest nicht durchgeführt werden. Für diese Patienten wurde die Diagnose Insektengiftallergie gestellt und eine SIT eingeleitet. Obwohl nur eine kleine Zahl an Patienten untersucht wurde, stellt das Immunoblotting in unklaren Situationen eine wertvolle Ergänzung für die Diagnosestellung und Therapieindikation von IgE-vermittelten Insektengiftallergien dar.

\section{Klinische Evaluation des PolyCheck ${ }^{\circledR}$-Systems zur Bestimmung von allergenspezifischem IgE}

\section{H. Wecker, B. Kubl, A. Laubert}

HNO-Klinik Universität Witten/Herdecke, Kath. Krankenhaus Hagen gGmbH

Hintergrund: Das seit kurzem auf dem Markt befindliche PolyCheck ${ }^{\circledR}$-System der Fa. milenia biotec ${ }^{\circledR}$ ist ein einfaches Verfahren zur quantitativen Bestimmung von allergen-spezifischem IgE. In dieser Untersuchung sollte das PolyCheck ${ }^{\circledR}-S y-$ stem verglichen werden mit dem etablierten CAP-System und die Ergebnisse evaluiert werden in Relation mit der klinischen Untersuchung.

Methodik und Ergebnisse: Zur Beurteilung der Messgenauigkeit des PolyCheck ${ }^{\circledR}$-Systems wurden die IgE-Konzentrationen in den Seren mit dem PolyCheck ${ }^{\circledR}$-System bestimmt und die Ergebnisse mit dem etablierten CAP-System der Fa. Pharmacia kontrolliert. Insgesamt korrelieren die erhaltenen IgEKonzentrationen gut zwischen den Systemen, wobei mit dem PolyCheck ${ }^{\circledR}$-System insgesamt niedrigere IgE-Konzentrationen gemessen werden.

Im Vergleich zu den Ergebnissen des Prick-Hauttestes zeigten die erhaltenen IgE-Konzentrationen eine gute Korrelation, wobei der Hauttest häufiger positive Ergebnisse ergab. Auch für die häufigsten positiven Einzelallergene D. pteronyssinus und Gräser ergaben sich im Prick-Hauttest wiederum häufiger positive Rekationen. Unter Berücksichtigung der Anamnese und der klinischen Beschwerden finden sich darunter jedoch auch falsch positive, nicht IgE-vermittelte Reaktionen.

Diskussion: Mit dem PolyCheck ${ }^{\circledR}$-System können mit geringem technischen Aufwand kostengünstig aussagekräftige Be- 